\title{
Gestor de contenidos de código abierto para archivos digitales sonoros que preservan materiales de investigación
}

\author{
Perla Olivia Rodríguez-Reséndiz* \\ Joséphine Simonnot** \\ Dafne Citlalli Abad Martínez*
}

Artículo recibido:

9 de agosto de 2018

Artículo aceptado:

14 de septiembre de 2018

Artículo de investigación

\section{RESUMEN}

Los institutos y centros de investigación generan una gran cantidad de documentos sonoros de origen digital como resultado de proyectos de investigación en ciencias sociales y humanidades. La acumulación de estos documentos digitales es un problema porque se carece de herramientas de preservación y el riesgo de pérdida es alto. Como una alternativa para preservar este tipo de documentos se presentan los alcances y funcionalidades de Telemeta, gestor de contenidos, para la preservación de contenidos sonoros.

\footnotetext{
* Instituto de Investigaciones Bibliotecológicas y de la Información Universidad Nacional Autónoma de México, México_perlaolivia@gmail.com

** Centre de Recherche en Ethnomusicologie, París, Francia dafne@iibi.unam.mx

josimonnot@gmail.com

INVESTIGACIÓN BIBLIOTECOLÓGICA, vol. 32, núm. 77, octubre/diciembre, 2018, México,
} ISSN: 2448-8321 pp. 101-115 
Palabras clave: Archivo Digital; Preservación Digital, Archivo Sonoro

Open source content managment for digital sound archives that preserve research materials Perla Olivia Rodríguez-Reséndiz, Joséphine Simonnot and Dafne Citlalli Abad-Martínez

\begin{abstract}
Institutes and research centers, through research projects in social sciences and humanities, generate a large number of digital sound documents. The accretion of these digital documents come with high risk of loss because of a lack of preservation tools. This paper describes the scope and functionalities of the Telemeta content manager as an alternative for preservation of sound files.
\end{abstract}

Keywords: Digital Archive; Digital Preservation; Sound Archive

\title{
INTRODUCCIÓN
}

C ha privilegiado el uso del término repositorio digital para denominar $\checkmark$ al sistema informático en el cual se almacena y organiza información de publicaciones científicas. El movimiento de Acceso Abierto impulsó la creación de repositorios digitales (Keefer, 2008) de materiales impresos (revistas, libros y documentos administrativos); tema que en la última década ha sido ampliamente documentado (Barnard, Delgado y Voutssas, 2014; Alcaráz et al., 2014; Lara, Serralde y Maniega, 2013, y Tramullas y Garrido, 2006). En contraste, las aportaciones para el diseño y creación de archivos digitales que preserven materiales sonoros son incipientes, si bien es evidente la necesidad de contar con herramientas de preservación digital de este tipo de documentos, que son frágiles y cuyo riesgo de pérdida es alto (IASA, 2011).

Las fonotecas, archivos sonoros de medios de comunicación, empresas de producción sonora, discográficas, así como centros de investigación, entre 
otros lugares, requieren garantizar la permanencia de contenidos digitales obtenidos como resultado de la digitalización o bien cuyo origen es digital y, para ello, deben contar con archivos digitales. La creación de archivos digitales para preservar colecciones sonoras es uno de los desafíos más complejos que deben encarar las instituciones de la memoria, sobre todo aquéllas que no cuentan con infraestructura, personal ni los recursos económicos de forma permanente.

Hace más de una década Bradley (2007) reconoció que era necesario desarrollar sistemas de almacenamiento y preservación en código abierto para que las instituciones pequeñas también preservaran sus colecciones. Se propuso crear un sistema simple y sustentable de código abierto como una alternativa de preservación digital (Bradley, 2007). Desde entonces, la comunidad científica y profesional ha mostrado interés en el uso del software en código abierto (open source) como una alternativa para preservación digital. A través del gestor de contenidos se controla el ciclo de vida de los objetos digitales (Crockett, 2016) y se articulan los procesos de identificación, organización, conservación y acceso en un archivo digital. La puesta en marcha de gestores de contenido es la primera etapa de creación de un archivo digital.

Este artículo presenta los alcances y funcionalidades de Telemeta como gestor de contenidos para la preservación de contenidos sonoros. Inicia con la metodología utilizada en la investigación y después se diserta sobre la relevancia de los archivos sonoros en la investigación científica. Más adelante, se examina el término archivo en los dominios analógico y digital. Continúa con la definición y descripción de los modelos de gestores de contenidos utilizados en los archivos sonoros y se presentan los resultados observados durante la primera etapa de instalación de Telemeta como gestor de contenidos. Para terminar, se ofrecen las conclusiones.

\section{Metodología}

Ante la acumulación de contenidos sonoros obtenidos como resultado de proyectos de investigación realizados en el Instituto de Investigaciones Bibliotecológicas y de la Información (IIBI) de la Universidad Nacional Autónoma de México (UNAM), se propuso la creación de un archivo digital para la preservación de estos materiales. Este proyecto se desarrolla en el marco de la colaboración de investigadores de la UNAM con el Centre de Recherche en Ethnomusicologie (CREM) de Francia y como parte del Proyecto PAPIIT IT- 400118 "Creación y desarrollo de archivos digitales multimedios (sonoros, audiovisuales y fotográficos) con open source. Una propuesta de transferencia 
tecnológica para la preservación digital de las colecciones de los pueblos originarios de México de la DGAPA UNAM".

Este artículo presenta los alcances y funcionalidades observados durante la primera etapa de instalación del gestor de contenidos de código abierto a partir del cual se establecerán las bases para la creación de un archivo digital que preserve las grabaciones de los pueblos originarios de México.

La metodología de este trabajo es teórica y aplicada. En cuanto a la investigación teórica se identificaron y analizaron publicaciones científicas para establecer el marco conceptual e histórico de los gestores de contenido como componentes de archivos digitales sonoros. Se describieron los modelos de gestores de contenidos digitales y se eligió trabajar con soluciones tecnológicas basadas en código abierto. La investigación aplicada se basó en observar, analizar y describir los alcances y funcionalidades del gestor de contenidos durante la primera etapa de instalación.

Los fondos iniciales incorporados en el gestor de contenidos materiales pertenecen a grabaciones de campo de dos grupos indígenas de México: los rarámuris y los purépechas. ${ }^{1}$ Estas grabaciones se obtuvieron de los proyectos PAPIIT-IN402016 "Preservación digital de documentos sonoros y audiovisuales de origen digital" y PAPIIT- IG400417 "Fortalecimiento, organización y preservación de la información originaria: bases para construir un modelo de biblioteca indígena de la comunidad purépecha del Municipio de Cherán", ambos auspiciados por la DGAPA (Dirección General de Asuntos del Personal Académico) de la UNAM.

\section{LOS ARCHIVOS SONOROS EN LA INVESTIGACIÓN CIENTÍFICA}

Los investigadores de disciplinas humanísticas y sociales fueron pioneros en utilizar las tecnologías para grabar y reproducir el sonido. Gracias al uso del fonógrafo en la investigación de campo grabaron música, danza, rituales y tradición oral como fuentes de información para el estudio de los fenómenos

1 Los rarámuris, también llamados tarahumaras, viven en condiciones de extrema pobreza entre las montañas de Chihuahua, en la Sierra Madre Occidental de México, en 17 municipios entre los que destacan por su densidad Guachochi, Norogachi, Urique, Batopilas, Balleza, Carichi, Guazapares, Bocoyna, Guerrero, Maguarichi, Nonoaca y Cuauhtémoc (CDI, 2017). Los púurbépecha, también denominados tarascos, viven en la región lacustre y montañosa del estado de Michoacán. En la actualidad los pururbépecha afrontan básicamente tres tipos de problemas: tala inmoderada de bosques, deterioro ambiental y seguridad social (Amézcua y Sánchez, 2015). Uno de los rasgos más destacados del pueblo p’urhépecha fue la instauración del Municipio Autónomo de Cherán en 2012, con lo que se pusieron "a prueba las viejas estructuras de la organización tradicional y permitió la emergencia de nuevos comportamientos organizacionales como la movilización de las mujeres de defensa frente a los embates criminales de la delincuencia organizada" (Amézcua y Sánchez, 2015: 139). 
sociales. Por ello, el desarrollo y florecimiento de la lingüística, etnomusicología y la antropología está vinculado al uso de las tecnologías para la grabación sonora (Schüller, 2008). Como resultado de la acumulación de materiales grabados en trabajos de investigación científica se fundaron los primeros archivos sonoros en el mundo: en 1899 en Viena, en 1900 en París y Berlín y en 1908 en San Petersburgo (Schüller, 2008).

Las posibilidades de grabación de campo se enriquecieron y diversificaron con la incorporación de la tecnología digital. La grabación sonora es, en la actualidad, un medio para generar recursos documentales para la investigación. Además, la grabación digital es una alternativa de salvaguarda frente al riesgo de pérdida de cuando menos el $50 \%$ de los idiomas que se hablan en la actualidad (Unesco, 2003b). Los investigadores de ciencias sociales y humanidades han emprendido, en atención a esta crítica situación, sendos proyectos de investigación para documentar la música, danzas, rituales, narraciones orales y paisaje sonoro, entre otras expresiones, de los pueblos originarios del mundo.

\section{DEL ARCHIVO SONORO ANALÓGICO AL DIGITAL}

A partir de 1980 y, en reacción a la "Recomendación sobre la salvaguarda y conservación del patrimonio audiovisual” (Unesco, 1980), los archivos sonoros fueron identificados como patrimonio intangible, legado, producto cultural y testimonio (ICA, 2018). Hasta el siglo pasado, el término archivo sonoro se utilizó para denominar el lugar, la institución de resguardo y el soporte en que fue registrado el sonido (Edmondson, 2018). El archivo sonoro como lugar de la memoria es el espacio donde se preservan las grabaciones sonoras, se protege la herencia audible de un pueblo y, coexisten, desde la perspectiva de Derrida (1995), el saber y el poder. Además, con esta expresión se nombra a la institución de la memoria en la cual se resguardan documentos sonoros (Edmondson, 2018). Los principales archivos sonoros son las fonotecas de alcance nacional, los centros de documentación musical, las fonotecas de radio, los archivos sonoros de investigación y especializados, entre otros. El vocablo también ha sido utilizado para hacer referencia al artefacto en el cual ha sido fijado el sonido (cilindros, discos, cintas de carrete abierto, casetes, discos compactos, digital audio tape, digital versatile disc, Blu-ray, linear tape open, disco duro, entre otros).

Los archivos sonoros comenzaron a utilizar soportes digitales en la década de 1980 (Prentice, 2016). El disco compacto fue el primer soporte digital que se empleó en los archivos sonoros como medio de resguardo (Bradley, 
2006). En algunos archivos, la noción archivo digital fue empleada para denominar a las copias de las grabaciones que se resguardaron en discos compactos.

La locución "archivo digital sonoro" se empleó por primera vez en la década de 1990, en la Conferencia Anual de la IASA (International Association of Sound and Audiovisual Archives / Asociación Internacional de Archivos Sonoros y Audiovisuales) organizada en Viena para hacer referencia a las soluciones tecnológicas necesarias para almacenar los contenidos obtenidos con la digitalización de colecciones analógicas (Schüller, 2008).

En los primeros años del siglo XXI, el archivo digital sonoro ha sido definido como un sistema de información y como un conjunto integrado de sistemas (Ojeda Castañeda, 2008) a través de los cuales se articulan los procesos documentales que intervienen durante el ciclo de vida de los objetos digitales, los roles, flujos de trabajo y usuarios que intervienen en la preservación digital (Rodríguez, 2016). El archivo sonoro es un término polisémico cuyos alcances y definiciones han cambiado a lo largo del tiempo. En este trabajo se emplea el término archivo digital sonoro para hacer referencia al sistema de información que vincula, a través del uso de herramientas tecnológicas, los procesos documentales, flujos de trabajo y usuarios que intervienen en el ciclo de vida para la preservación digital.

\section{Gestión DE COLECCiONeS Digitales}

El término gestión de contenidos tiene diferentes usos y su concepción ha cambiado a lo largo del tiempo. Se empleó para denominar a los programas informáticos de edición colaborativa de sitios web. También se ha utilizado para nombrar a las herramientas, métodos y procesos de la gestión documental tradicional (Eíto-Brun, 2013: 378). De forma más reciente se define como la "integración progresiva de las tecnologías para gestionar información y datos no estructurados" (Eíto-Brun, 2013: 378).

En la década de 1990 se instalaron los primeros sistemas de gestión de colecciones digitales con funciones de ingesta, selección, descripción, almacenamiento, vinculación, base de datos para catalogar, así como herramientas de búsqueda y recuperación de materiales (Monson, 2017 y Rodríguez Pallares, 2015). Los gestores de contenidos son una nueva herramienta tecnológica que surgió con la digitalización (Muñoz, Meana y Sáez, 2014) y ante la necesidad de manejar y administrar información y datos no estructurados. Los sistemas de gestión se han denominado con diferentes nombres: Sistema de gestión de contenidos, Sistema de gestión de activos digitales, 
Repositorio digital y Sistema de gestión y almacenamiento masivo digital. En el ámbito de los archivos sonoros, las soluciones pioneras fueron los Sistemas de gestión y almacenamiento masivo digital (SGAMD). Estos fueron concebidos para almacenar los contenidos obtenidos después de la digitalización, obtener copias automatizadas y apoyar la gestión de contenidos a largo plazo (Murray, 2014); con ello la forma tradicional de trabajo en los archivos sonoros se transformó (Cavaglieri, 2009) en la primera década del siglo XXI. Los SGAMD cohabitaron con el archivo analógico. En un principio, no se dimensionó el alcance que éstos podrían tener; sin embargo, de forma progresiva ganaron importancia dentro de la institución.

El gestor de contenidos es un componente del archivo digital. De acuerdo con el Open Archival Information System (OAIS) es el conjunto de servicios y funciones necesarias para generar, mantener y hacer accesibles la información descriptiva, que identifica y documenta los fondos, los datos administrativos del catálogo y el registro estadístico de los datos contenidos (IASA, 2011). "La gestión y administración de datos resulta decisiva en un sistema de archivo sostenible, puesto que garantiza que los ficheros preservados y accesibles puedan ser adecuadamente hallados e identificados" (IASA, 2011: 126).

Monson (2017) ha señalado que existen tres modelos de gestores de contenidos digitales: diseño propio, propietario o comercial y de código abierto. El diseño propio es el desarrollo basado en las características y necesidades de las instituciones. Las instituciones que cuentan con un amplio presupuesto y un sólido equipo de tecnologías de información pueden poner en marcha un sistema de gestión de contenidos de esta naturaleza.

Los sistemas propietarios o comerciales se asientan en la venta de licencias, el pago sistemático de servicios de mantenimiento y actualizaciones del uso de software. Los clientes cuentan con soporte técnico. Esta alternativa es relativamente fácil de poner en marcha en instituciones que no disponen de personal en el área de tecnología. El riesgo más grande de esta modalidad es que se interrumpa el suministro de recursos económicos y con ello se limite la continuidad del servicio. Este tipo de software supone "gastos continuos y el peligro de quedar encasillados en un sistema propietario del que es difícil escapar" (IASA, 2011:133).

Las soluciones de código abierto generalmente están disponibles en internet de forma gratuita. Este tipo de opciones "tienen a su favor que la adhesión a estándares y marcos abiertos que permiten la extracción o migración del contenido" (IASA, 2011: 133). Bradley (2007) fue pionero en proponer el uso del software de código abierto, de bajo costo, para crear sistemas de archivo digital abierto que conserven objetos en diferentes formatos (audio, 
texto, imagen en movimiento y fotografía). Este tipo de software es utilizado, sobre todo, en el sector cultural y educativo. Dependiendo de la comunidad de usuarios, el software puede ser modificado, enriquecido y contar con nuevos desarrollos y funcionalidades (Monson, 2017). La desventaja es que, a pesar de contar con ayuda de las comunidades de código abierto, el mantenimiento depende del usuario. Para afrontar esta situación pueden contratarse proveedores comerciales que ofrezcan asistencia técnica (IASA, 2011). En la Tabla 1 se ofrece una relación de gestores de contenidos digitales de código abierto cuya configuración admite la incorporación de formatos de preservación (abiertos y sin compresión) de documentos digitales sonoros.

En los últimos años, la línea entre los sistemas propietarios y de código abierto ha empezado a ser menos clara. "Una tercera parte de los vendedores ahora ofrecen soporte comercial a las instituciones que usan open source. El rango de servicios que ofrecen comprende desde soporte técnico por hora, hasta un paquete de servicios que incluye almacenamiento en la nube y mantenimiento. Con ello, es posible combinar los beneficios de las soluciones propietarias con el open source en una solución de software" (Monson, 2017: 113).

\begin{tabular}{|c|c|}
\hline Sistemas open source & Año de creación \\
\hline Greenstone & Finales 1990 \\
\hline Dspace & 2002 \\
\hline Fedora & Empezó en 1997 y se liberó en 2003 \\
\hline Islandora & 2006 \\
\hline Hydra & 2008 \\
\hline Telemeta & Telemeta \\
\hline
\end{tabular}

Tabla 1. Gestores de contenidos digitales de código abierto Fuente: elaboración propia

\section{Resultados}

El archivo digital, como se ha señalado, es un sistema de información cuya arquitectura tecnológica se sustenta en el gestor de contenidos y el almacenamiento digital distribuido. En este trabajo se aborda solamente el gestor de contenidos como herramienta para la organización, identificación, conservación, acceso y reaprovechamiento documental. Se eligió trabajar con Telemeta por ser un software creado en código abierto y porque fue concebido para que los investigadores de las ciencias sociales, sobre todo los antropólogos, 
etnomusicólogos y lingüistas, puedan preservar los materiales que graban como resultado de sus trabajos de investigación de campo.

El desarrollo de Telemeta comenzó en 2007, como parte de la colaboración entre la compañía Parisson y el CREM y se puso en marcha en 2011 (Fillon y Pellerin, 2017). Este software está disponible bajo la Licencia de Software Libre GNU Affero General Public License v3.0 y es una herramienta para gestión de contenidos multimedia. Telemeta ha sido definida como una plataforma de audio web escalable, que permite realizar copias de seguridad, indexar, transcodificar, analizar, compartir y visualizar cualquier formato de archivo de audio o video digital de estándares abiertos de la web (Fillon et al., 2014).

Derivado del proceso de instalación de Telemeta se han identificado los siguientes alcances y funcionalidades para la construcción de un archivo digital que preserve grabaciones sonoras de pueblos originarios de México:

a) Conservación. El gestor de contenidos fue creado para conservar y dar acceso a materiales sonoros creados en formatos de preservación, es decir, abiertos y sin compresión. Esta funcionalidad es la primera que fue considerada en la puesta en marcha de esta herramienta; no obstante, también se pueden almacenar materiales audiovisuales, fotográficos e iconográficos (imágenes digitalizadas, escaneos de folletos y notas de campo, etc.), hipervínculos e información biográfica sobre el recolector (Fillon et al., 2014 y Khoury y Simonnot, 2014). Esta alternativa es de gran apoyo para el trabajo de investigación puesto que, además de grabaciones sonoras, durante el trabajo de campo se recopilan grabaciones audiovisuales, fotografías y notas de campo que también deben ser preservadas.

b) Acceso y consulta. Los materiales pueden ser consultados y descargados en formatos sin compresión como WAV, o bien de compresión, como FLAT, OGG, MP3. El acceso para consulta o descarga de los materiales se configura de acuerdo con los permisos que tienen los usuarios y con base en los derechos de autor de cada una de las grabaciones. Es preciso señalar que en la creación del archivo digital se busca privilegiar el acceso abierto a partir de contar con el consentimiento de los pueblos originarios. En la Figura 1 se muestra la interfaz de acceso al gestor de contenidos. 


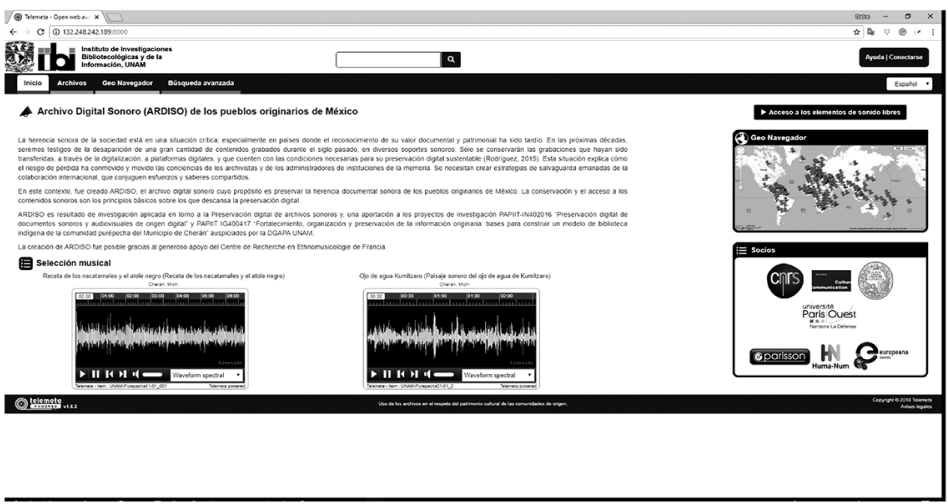

Figura 1. Interfaz de acceso de Telemeta

c) A través del gestor de contenidos se puede ofrecer el acceso in situ o bien en línea a través de la página web. Para el acceso a las colecciones, Telemeta contiene reproductores de audio y video a través de Timeside (audio processing framework). Este procesador puede mostrar el sonido gráficamente en forma de onda y como espectrograma.

d) Identificación. La estructura de los metadatos es interoperable, se basa en los protocolos Dublin Core y OAI-PMH (Open Archives Initiative Protocol for Metadata Harvesting). Se pueden incluir detalles sobre el lugar donde se realizó la grabación, los instrumentos musicales, la población, el título de la pieza musical, los elementos culturales relacionados con el elemento musical, el depositante, el coleccionista, el año de la grabación y el año de publicación de documentos que describen el trabajo (Fillon et al., 2014). Si bien la estructura de Telemeta permite mantener los metadatos separados de sus objetos digitales, éstos se resguardan de manera predeterminada en una misma ubicación física. Para tener un almacenamiento distribuido, es necesario reconfigurar el sistema.

e) El gestor de contenidos tiene herramientas de edición social. Es una plataforma colaborativa (Fillon y Pellerin, 2017) a través de la cual usuarios especializados como los investigadores pueden establecer marcas y anotaciones en las grabaciones y compartirlas con sus colegas. Estas anotaciones son accesibles desde la página web del archivo de sonido y están indexadas a través de la base de datos (Fillon et al., 2014). Con ello, se pueden enriquecer los metadatos obtenidos durante la grabación de campo. 
f) Organización. La organización intelectual de los materiales tiene cuatro niveles: fondos, corpus, colecciones e ítems. Estos niveles se pueden adecuar dependiendo de las necesidades de cada archivo. Los fondos iniciales que se incorporaron son Rarámuris y Purépechas. El fondo Rarámuri está formado por dos corpus: Continente Rojo y Semana Santa Rarámuri. Continente Rojo agrupa un conjunto de colecciones de las fiestas tradicionales, danzas, música, rituales y entrevistas a diversos pobladores de la comunidad rarámuri que, desde el año 2010, grabó la antropóloga francesa Sylvie Marchand. Los materiales sonoros (entrevistas, paisajes sonoros, danzas, música y rituales) fueron registrados con tecnología digital en formato WAV de 24 bits/ 48 khz. Además, se cuenta con grabaciones audiovisuales (entrevistas, fiestas tradicionales, rituales, danzas, etc) grabadas en formato AVCHD y MOV. Las producciones poseen alta calidad artística y técnica. Los materiales grabados por Marchand son documentos de origen digital, que hasta ahora no han sido preservados en alguna institución de la memoria en México. Por ello, se le propuso a la investigadora participar en este proyecto de investigación destinado a crear estrategias de salvaguarda de esta herencia documental. Por su parte, el corpus Semana Santa Rarámuri reúne grabaciones sonoras, audiovisuales y fotografías del trabajo de campo realizado por investigadores del IIBI en la comunidad de Norogachi, Chihuahua, en 2016.

En el fondo Purépecha se incorporó el corpus "Cien sonidos del paisaje sonoro purépecha" que reúne grabaciones sonoras de personajes emblemáticos que dan cuenta de la historia y tradiciones de Cherán, fiestas tradicionales, narración de tradiciones, sonoridad de las lenguas habladas en la comunidad, entornos naturales, oficios tradicionales (zapateros, vendedores de frutas, etc), música tradicional, entre otros sonidos de relevancia para la comunidad. Los sonidos grabados fueron identificados por parte de la comunidad e incorporados al archivo digital para su resguardo. Estos materiales a su vez formarán parte de la biblioteca modelo de Cherán.

g) Para la instalación del gestor de contenidos digitales se requieren conocimientos de administración de sistemas operativos en Linux. El gestor, desde su versión 1.6, incorpora un paquete de Docker que asocia todas las aplicaciones y módulos necesarios para llevar a cabo la instalación. La estructura del software es Modelo-Vista-Controlador que separa la interfaz del usuario, la gestión de las solicitudes y la administración de los datos contenidos. Utiliza como lenguaje de programación Phyton, el framework Django e incorpora MySql como 
motor de la base de datos. El diseño de Telemeta incluye tecnologías compatibles con HTML5, característica que lo hace funcional para diferentes navegadores web.

\section{Conclusiones}

Los científicos sociales y de humanidades registran mediante tecnologías digitales materiales sonoros que contribuyen al estudio y a la solución de problemas sociales. Una de estas vertientes es la documentación de las lenguas originarias que se encuentran en riesgo de desaparecer. La acumulación de estas grabaciones trae consigo grandes desafíos. Se necesita conservar y dar acceso a grabaciones sonoras y materiales asociados (videos, fotografías y notas de campo), y para ello es necesario crear archivos digitales.

El archivo digital sonoro es el sistema de información que vincula, a través del uso de herramientas tecnológicas, los procesos documentales, flujos de trabajo y usuarios que intervienen en el ciclo de vida para la preservación digital. El gestor de contenidos es la herramienta base para la preservación digital a largo plazo. La instalación de gestores de contenido de código abierto es una alternativa para la preservación de los materiales que han sido grabados como resultado de la actividad científica.

La puesta en marcha de Telemeta como gestor de contenidos de código abierto sienta las bases para la creación de un archivo digital sonoro de acceso abierto y es resultado del proyecto de investigación e innovación tecnológica basado en la colaboración de investigadores de México y de Francia.

La colaboración es necesaria en la preservación digital. La tecnología compartida, el almacenamiento distribuido y las estrategias de preservación conjunta son acciones para salvaguardar contenidos digitales, especialmente para regiones como América Latina, donde los recursos económicos son escasos y no se asignan de forma sistemática, son muy pocas las investigaciones que se realizan en este campo y donde aún es necesario formar y actualizar a los profesionales que trabajan en los archivos sonoros.

\section{REFERENCIAS}

Alcaráz, Rubén, Sergi Montes, Lluis Vicente, Merche Blanco, Elena Álvaro, Laia Amorós, Pablo Casas, Laura Nieto y Marc Reig. 2014. "El Archivo de la Palabra: contexto y proyecto del repositorio audiovisual del Ateneu Barcelonés”. BID Textos Universitaris de biblioteconomía i documentació 33 (Barcelona, España). 
Amézcua, J. y G. Sánchez. 2015. Pueblos indígenas de México en el siglo XXI. P'urbépecha. México: Comisión Nacional para el Desarrollo de los Pueblos Indígenas (CDI).

Barnard, A., A. Delgado y J. Voutssas. 2014. Los archivos digitales. Una visión integradora. México: Archivo Histórico Benemérita Universidad Autónoma de Puebla.

Bradley, K. 2006. Riesgos asociados con el uso de los discos compactos (CDs) y videodiscos (DVDs) como medios confiables de almacenamiento para colecciones de archivo. México: Fonoteca Nacional.

Bradley, K. 2007. Hacia un sistema de almacenamiento y preservación en código abierto. Recomendaciones respecto a la implementación de un sistema de preservación de archivos digitales y temas en torno al desarrollo de software. México: Unesco.

CDI (Comisión Nacional para el Desarrollo de los Pueblos Indígenas). 2017. Indicadores socieconómicos de los pueblos indígenas de México. México. Fecha de consulta: 12 de marzo de 2018 https://www.gob.mx/cdi/articulos/indicadores-socioeconomicos-de-los-pueblos-indigenas-de-mexico-2015-116128?idiom=es

Cavaglieri, S. 2009. "Criteria to consider in the definition of digital mass storage systems", en La salvaguarda del patrimonio sonoro y audiovisual: un reto mundial. Memorias del Cuarto Seminario Internacional de Archivos Sonoros y Audiovisuales, Perla Rodríguez (coord.), 151-164. México: CONACULTA, Fonoteca Nacional de México.

Crockett, Margaret. 2016. The no-nonsense guide to archives and recordkeeping. Londres: Facet Publishing.

Derrida, J. 1995. Archive Fever. Chicago: The University of Chicago Press.

Edmondson, R. 2018. Filosofía y principios de los archivos audiovisuales, 3a. ed. México, Unesco, UNAM-UASLP.

Eíto-Brun, Ricardo. 2013. "Madurez de la gestión de contenidos, ¿sinónimo de desgaste o de oportunidades?”. El profesional de la información 22 (5) (septiembre-octubre): $377-380$.

Fillon, Thomas, Josephine Simonnot, Marie-France Mifune, Stéphanie Khoury, Guillaume Pellerin, Maxime Le Coz, Estelle Amy De la Bretèque, David Doukhan y Dominique Fourer. 2014. "Telemeta: An open-source web framework for ethnomusicological audio archives management and automatic analysis", Proceedings of the 1st International Workshop on Digital Libraries for Musicology, ACM: 1-8.

Fillon, T. y G. Pellerin. 2017. "A collaborative web platform for sound archives management and analysis", Proceedings of $3 r d$ Web Audio Conference, London.

ICA (International Council on Archives). 2018. ¿Qué es un archivo? Fecha de consulta: 22 de marzo de 2018. https://www.ica.org/es/\%C2\%BFqu\%C3\%A9-son-los-archivos

IASA (International Association of Sound and Audiovisual Archives). 2011. Directrices para la producción y preservación de objetos digitales de audio. IASA-TC04. España: AEDOM.

Keefer, A. 2008. "Los repositorios digitales universitarios y los autores”. Revista Anales de Documentación (España): 205-214.

Khoury, S. y J. Simonnot. 2014. "Applications and implications of digital audio databases for the field of ethnomusicology: A discussion of the CNRS - Musée de l'Homme sound archives". First Monday 19 (10-6). doi: http://dx.doi. org/10.5210/fm.v19i10.5554 
Lara, P., E. Serralde y E. Maniega. 2013. "Evolución de los repositorios documentales. El caso SOCIALNET”. El Profesional de la Información 22 (5) (septiembre-octubre).

Ojeda Castañeda, G. 2008. Los archivos audiovisuales en las redes digitales de comunicación para la educación y la cultura. Informe de Investigación y Documentación Analítica. Serie de Informes CNICE. Ministerio de Educación y Ciencia de España. Fecha de consulta: 20 de mayo de 2018. http://ares.cnice.mec.es/informes/13/contenido/34.htm

Prentice, Will. 2016. "Digital preservation of audio content", en Mananging digital cultural objects. Chicago: Neal Schuman.

Monson, J. 2017. Getting started with digital collections. Scaling to fit your organization. Chicago: American Library Association.

Muñoz, P., S. Meana y S. Sáez. 2014. "Cinco años de experiencia digital en los Servicios Informativos de TVE: una nueva gestión de contenidos”. El profesional de la información 23 (1) (enero- febrero): 72-79.

Murray, K. 2014. Audio for Eternity: Schüller and Häfner Look. Back at 25 Years of Change. Fecha de consulta: 20 de marzo de 2018. https://blogs.loc.gov/thesignal/2014/11/audio-for-eternity-schuller-and-hafner-look-back-at-25-years-of-change/

Rodríguez Pallares, M. 2015. "Integración y visibilidad de la gestión de contenidos, dos retos por alcanzar en el modelo de comunicación radiofónica digital. Análisis de los casos de SER, COPE y RNE". Revista General de Información y Documentación 25 (1): 191-213. doi: http://dx.doi.org/10.5209/rev_RGID.2015.v25. n1.48987

Rodríguez, P. 2016. "El OAIS en la preservación digital de archivos sonoros". Investigación Bibliotecológica: archivonomía, bibliotecología e información 30 (70): 197220. http://dx.doi. org/10.1016/j.ibbai.2016.10.009

Schüller, D. 2008. Audiovisual research collections and their preservation. Amsterdan European Comission on Preservation and Acces.

Tramullas, J. y P. Garrido. 2006. "Software libre para repositorios institucionales: propuestas para un modelo de evaluación de prestaciones". El profesional de la información 15 (3) (mayo-junio).

Unesco. 1980. Recomendación sobre la salvaguardia y la conservación de las imágenes en movimiento. Conferencia General de la Organización de las Naciones Unidas para la Educación, la Ciencia y la Cultura. Fecha de consulta: 30 de mayo de 2018. http://portal.unesco.org/es/ev.php-URL_ID=13139\&URL_DO=DO_TOPIC\&URL_SECTION=201.html

Unesco. 2003a. Directrices para la preservación del patrimonio digital. París. Fecha de consulta: 16 de marzo de 2018. http://unesdoc.unesco.org/images/0013/001300/130071s. pdf

Unesco. 2003b. Vitalidad y peligro de desaparición de las lenguas. París: Unesco, Grupo Especial de Expertos sobre Lenguas en Peligro de Extinción. 
Proyecto PAPIIT IT- 400118 "Creación y desarrollo de archivos digitales multimedios (sonoros, audiovisuales y fotográficos) con open source. Una propuesta de transferencia tecnológica para la preservación digital de las colecciones de los pueblos originarios de México de la DGPA UNAM”.

Para citar este texto:

Rodríguez-Reséndiz, Perla Olivia, Joséphine Simonnot y Dafne Citalli Abad-Martínez. 2018. "Gestor de contenidos de código abierto para archivos digitales sonoros que preservan materiales de investigación". Investigación Bibliotecológica: archivonomia, bibliotecología e información 32 (77): 101-115.

http://dx.doi.org/10.22201/iibi.24488321xe.2018.77.58005 